\title{
Planar Sensor for Material Characterization Based on the Sierpinski Fractal Curve
}

\author{
P. H. B. Cavalcanti Filho, ${ }^{1}$ J. A. I. Araujo, ${ }^{1}$ M. R. T. Oliveira, ${ }^{1}$ M. T. de Melo, ${ }^{1}$ M. S. Coutinho, ${ }^{1}$ \\ L. M. da Silva, ${ }^{1}$ and I. Llamas-Garro ${ }^{2}{ }^{2}$ \\ ${ }^{1}$ Departamento de Eletronica e Sistemas, Universidade Federal de Pernambuco (UFPE), Recife, Brazil \\ ${ }^{2}$ Centre Tecnologic de Telecomunicacions de Catalunya (CTTC/CERCA), Castelldefels, Barcelona, Spain \\ Correspondence should be addressed to I. Llamas-Garro; ignacio.llamas@cttc.es
}

Received 4 May 2020; Revised 29 September 2020; Accepted 16 November 2020; Published 3 December 2020

Academic Editor: Davide Palumbo

Copyright ( 2020 P. H. B. Cavalcanti Filho et al. This is an open access article distributed under the Creative Commons Attribution License, which permits unrestricted use, distribution, and reproduction in any medium, provided the original work is properly cited.

\begin{abstract}
This paper presents a planar and compact microwave resonator sensor to characterize materials. The geometry of the resonator is based on the Sierpinski fractal curve and has four poles in the frequency range from $0.5 \mathrm{GHz}$ to $5.5 \mathrm{GHz}$. Any of the four poles can be used to measure samples with low permittivity values, where the first pole is suitable for samples with high permittivity values. The sensitivity of the poles and return losses of the sensor are presented and obtained using a full-wave 3D simulator software. The device is manufactured and validated through a comparison between simulated and measured results.
\end{abstract}

\section{Introduction}

The microwave region of the electromagnetic spectrum is commonly used for dielectric material characterization, by sensing through electromagnetic radiation. Applications include agricultural and food product testing $[1,2]$, industrial material measurements [3-6], in addition to chemical and biological liquid tests [7].

Sensors operated by measuring the attenuation or reflection coefficient, when in contact or in proximity to a measured sample, can be used to measure the permittivity of dielectric materials.

Microwave sensors have been widely used in different types of applications for the characterization of dielectric materials [8]. Microstrip devices are composed of a conductive strip, a dielectric substrate, and a ground plane [9]. Microstrip sensors use transmission and reflection signals for material characterization [10].

A new planar sensor with multiband behavior is presented in this work. The proposed device is designed and developed using a resonator with the Sierpinski curve geometry, for the characterization of dielectric materials. The sensor is designed to operate in the frequency range from $0.5 \mathrm{GHz}$ to $5.5 \mathrm{GHz}$.
The proposed sensor operates at multiple frequency bands, thus, a variety of materials with different permittivity values can be measured using the four resonant frequencies simultaneously.

\section{Sierpinski Curve Fractal Geometry}

The term fractal was introduced by the French mathematician Benoît Mandelbrot in 1975 and derived from the Latin fractus, adjective of the verb frangere, which means to break, to create irregular fragments, or to fragment. A fractal has a shape whose parts resemble the whole structure, thus as the scale of observation increases or decreases, its shape is unchanged, remaining identical or very similar to the original structure. The fractal geometry describes certain phenomena of nature or intricate objects, where Euclidean geometry (points, lines, and circles) is not able to describe, due to the simplicity of its forms [11].

The Sierpinski curve is named after the Polish mathematician Waclaw Sierpinski, who originally devised it around 1912 and is much less known compared to other fractal objects created by Sierpinski and his coworkers, such as the Sierpinski gasket or the Sierpinski carpet [12]. It is a continuous curve that fills the whole plane without intersecting lines; therefore, it is a space-filling curve with similar and 
simple auto-geometry. Figure 1 shows the first three fractal iterations of the Sierpinski curve. Because it is a spacefilling curve, as the fractal iteration order increases, the patch or microstrip transmission line area increases, but without increasing the total area occupied by the structure. In view of this, in this work, the structure is used for sensor applications since the structure is sensible due to the intrinsic high capacitance of the structure [13].

If we imply $x_{n}, k_{n}$ as the total length and area of the Sierpinski curve with different iterations, where $n$ is the iteration number, and consider $k$ as the side length of the original large square, the succeeding cases can be verified [14]:

$$
\begin{gathered}
x_{1}=\left(1+\frac{3 \sqrt{2}}{2}\right) k, x_{\infty} \rightarrow \infty, \\
K_{1}=\frac{11 k^{2}}{32}, \\
K_{\infty}=\frac{5}{12} k^{2} .
\end{gathered}
$$

From equation (3), the limit of the Sierpinski curve is infinitely long and fills an area of 5/12 of the square. The Hausdorff dimension is admitted as an effective way to precisely measure the dimension of highly asymmetrical sets. From the recursion shown in Figure 1, it is apparent that the Sierpinski curve consists of four replicas, each analogous to the whole, scaled by a factor of $1 / 2$. Thus, for Sierpinski curves, the Hausdorff dimension is equivalent to the similarity dimension and can be calculated using the following equation $[15,16]$ :

$$
H_{d}=S_{d}=-\frac{\ln N}{\ln s(N)}=-\frac{\ln 4}{\ln (1 / 2)}=2,
$$

where $H_{d}$ is the Hausdorff dimension, $S_{d}$ is the similarity dimension, $N$ is the number of self-similar copies, and $s(N)$ is the scale factor. Mathematically, the result anticipates that the Sierpinski curve will completely occupy the whole large square at the limit, despite its path being one-dimensional [17].

\section{Sierpinski Curve Sensor}

The first iteration $(n=1)$ of the Sierpinski curve is used to form a resonator as shown in the center of Figures 2(a) and 2(b). The shaded box placed over the resonator in Figure 2(a) represents the sample. The choice of $n=1$ was based on the operating frequency range, keeping low manufacturing complexity and high fabrication yield. The sensor with $n=1$ showed good sensitivity when measuring the samples of interest, higher values of $n$ would lead to greater manufacturing complexity, as shown in [13], and more precise designs can be implemented using laser machining or lithography. In this work, the interest is to obtain good sensitivity, adequate to measure samples of well-known materials to validate the sensor experimentally.

The fractal resonator occupies an area of $800 \mathrm{~mm}^{2}$ $(28.3 \times 28.3 \mathrm{~mm})$. The sensor is designed in microstrip form,

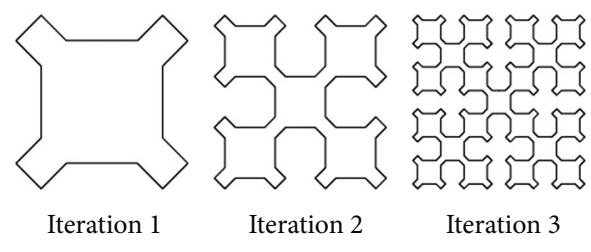

Figure 1: First three iterations of the Sierpinski curve fractal.

with substrate thickness of $1.27 \mathrm{~mm}$, relative dielectric constant of 10.2 , and total size of $100 \times 66.7 \mathrm{~mm}$. The line widths of the fractal resonator are $0.71 \mathrm{~mm}$ and $1 \mathrm{~mm}$, as shown in Figure 2(b). The resonator is coupled to two input/output microstrip transmission lines having a width of $0.6 \mathrm{~mm}$ and a characteristic impedance of $50 \mathrm{Ohms}$.

The resonator geometry presents a long effective length which provides a multiband response, and the capacitance created by line proximity increases sensor sensitivity. The resonator microstrip width changes are added to match the lines of the resonator; the structure is symmetrical and has thicker lines where the input feed line is attached, designed for fabrication robustness. Resonator line widths are chosen after optimization through simulations focused on high sensitivity, linked to easy and robust manufacturing.

\section{Simulation, Results, and Discussion}

The simulation results regarding return loss and sensitivity of the sensor are presented and analyzed in this section.

Figure 3 shows the return losses of the fractal sensor without sample obtained by CST Microwave Studio, for the frequency range between 0.5 and $5.5 \mathrm{GHz}$. The sensor has four resonances at $1.24,2.29,3.74$, and $4.94 \mathrm{GHz}$, with $-10 \mathrm{~dB}$ bandwidths of $240,200,160$, and $200 \mathrm{MHz}$, respectively. The resonance frequencies have very similar characteristics, such as a bandwidth of approximately $200 \mathrm{MHz}$ and return loss better than $-35 \mathrm{~dB}$ for the four resonance frequencies of interest for sensing.

The values of the reflection coefficient were sufficient for sensor operation and are suitable for material characterization; therefore, the transmission data was not collected during measurements.

Materials have a specific dielectric permittivity at RF and microwave frequencies [18]; thus, a sample with a parallelepiped shape and dimensions of $38.3 \times 38.3 \times 3 \mathrm{~mm}$ is placed over the area occupied by the fractal resonator, where the highest concentration of electric field is found, and the permittivity is then varied from 1 to 100 . With this configuration, any sample material can be simulated, through the variation of its permittivity.

In the microstrip transmission line structure, the field is more concentrated in the substrate, but still a weak electric field above it exists. The calculations involving microstrip structures use the effective permittivity, to take into account the permittivity of the substrate and the medium above the substrate, which is usually air [19]. As a result, if any material is placed on top of the resonator, the effective permittivity will vary, and consequently, the resonant frequency will shift. 


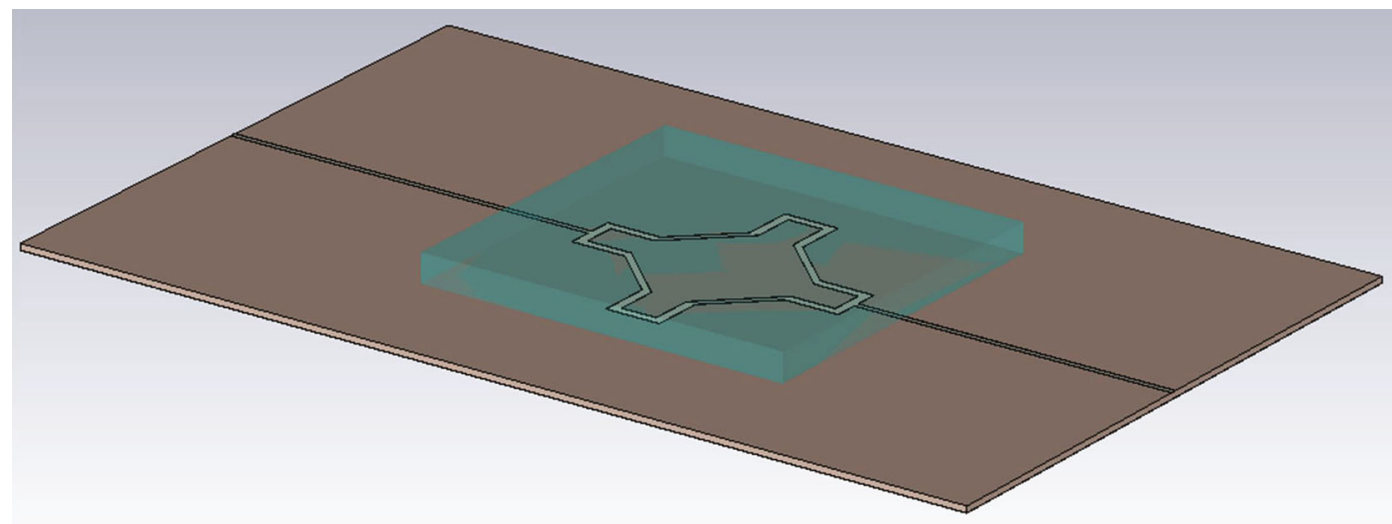

(a)

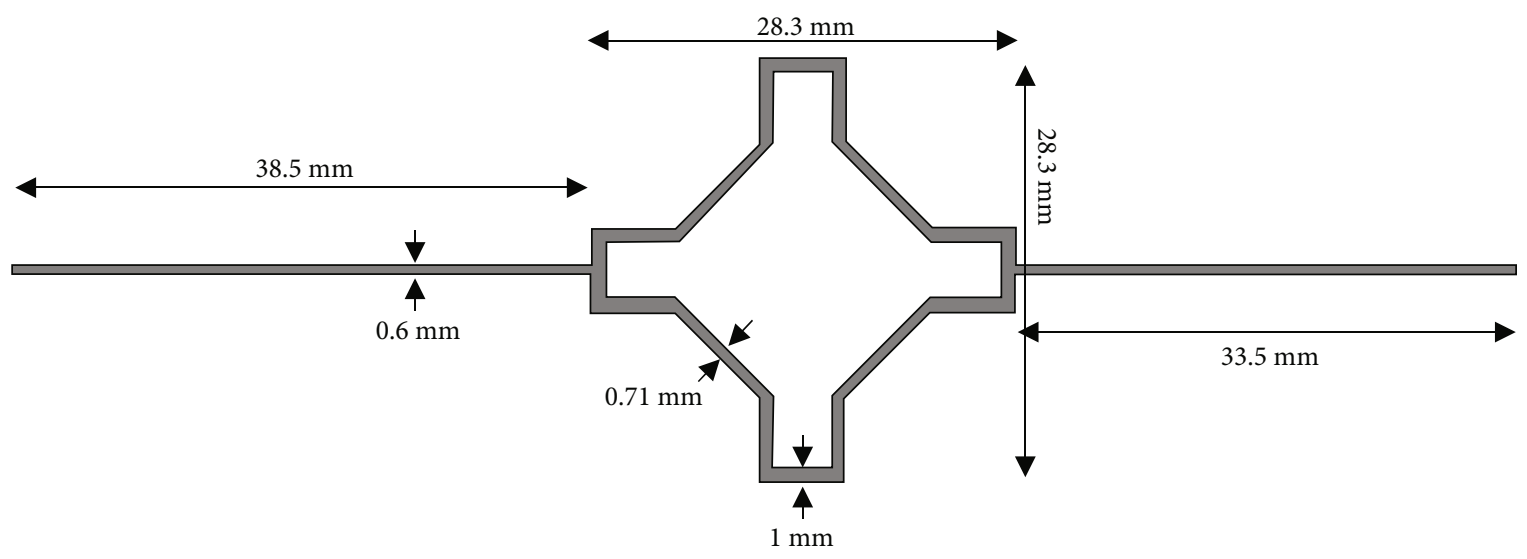

(b)

FIGURE 2: Proposed fractal sensor: (a) sensor with sample on top; (b) sensor dimensions.

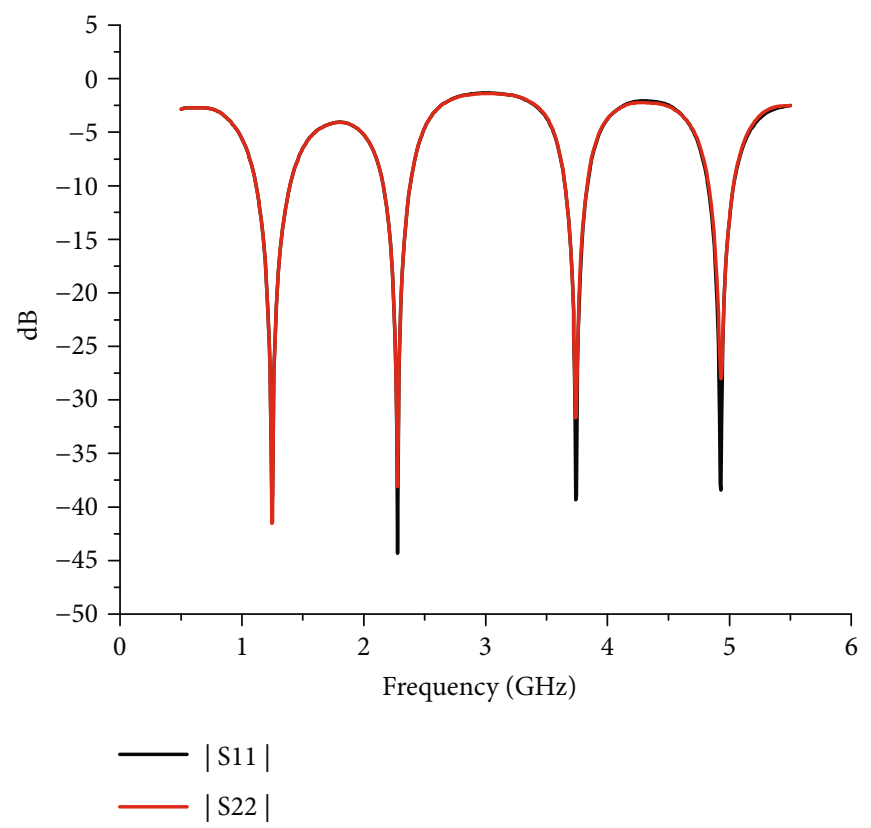

FIGURE 3: Return losses of the proposed fractal sensor without sample. 


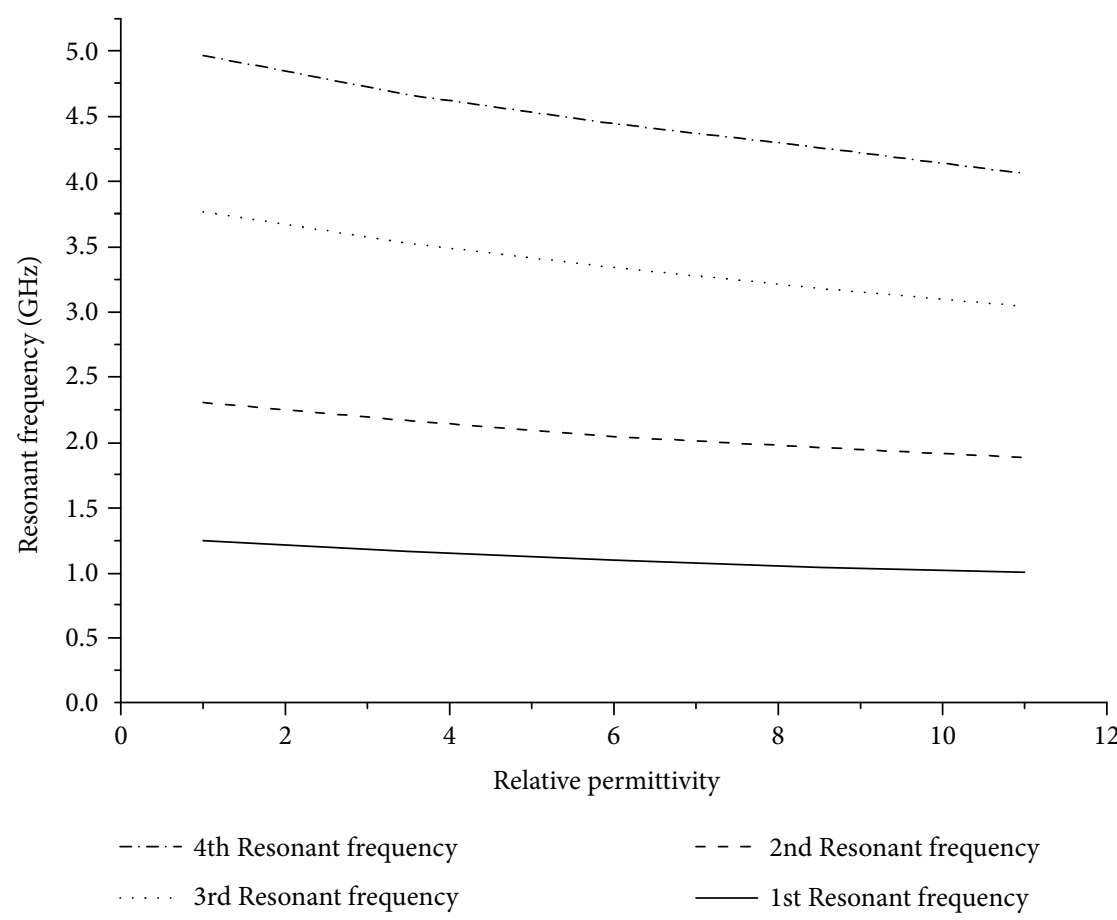

FIgURE 4: Resonant frequency as a function of sample permittivity (between 1 and 10) for the four sensor poles.

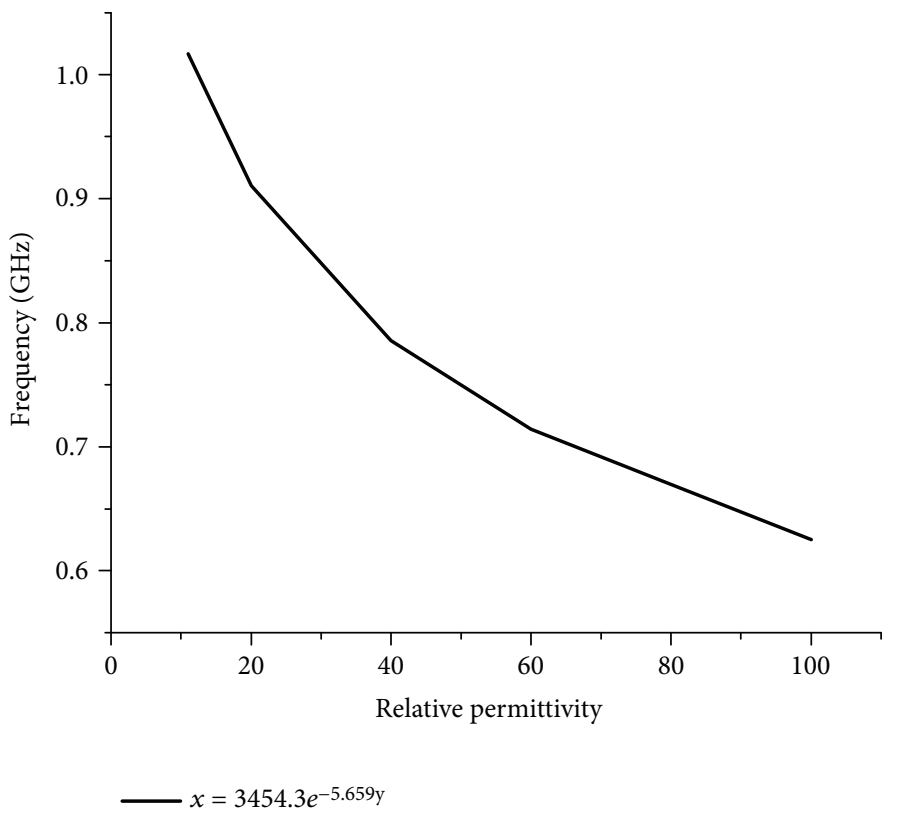

Figure 5: Sensitivity of the first resonant frequency of the fractal sensor for a permittivity range between 10 and 100.

Figure 4 shows the variation of resonant frequencies versus sample permittivity for all four resonances studied. The sensitivity of the first, second, third, and fourth resonances is 23.1, 39.9, 68.7, and 84.8, respectively. Equations (5)-(8) provide expressions that associate the resonant frequency of each pole with the permittivity of the samples. Therefore, by obtaining the four measured resonant frequencies $\left(f r_{n}\right)$ of the sensor-sample system, it is possible to calculate the permittivity $\left(\varepsilon_{r}\right)$ of the samples through these equations:

$$
\begin{gathered}
\varepsilon_{r}=-42.343 f r_{1}+53.347, \\
\varepsilon_{r}=-24.614 f r_{2}+56.875, \\
\varepsilon_{r}=-14.376 f r_{3}+54.444, \\
\varepsilon_{r}=-11.675 f r_{4}+58.245 .
\end{gathered}
$$

The four poles can be used to measure the samples with permittivity values between 1 and 10. The first resonant frequency is chosen to measure samples with 


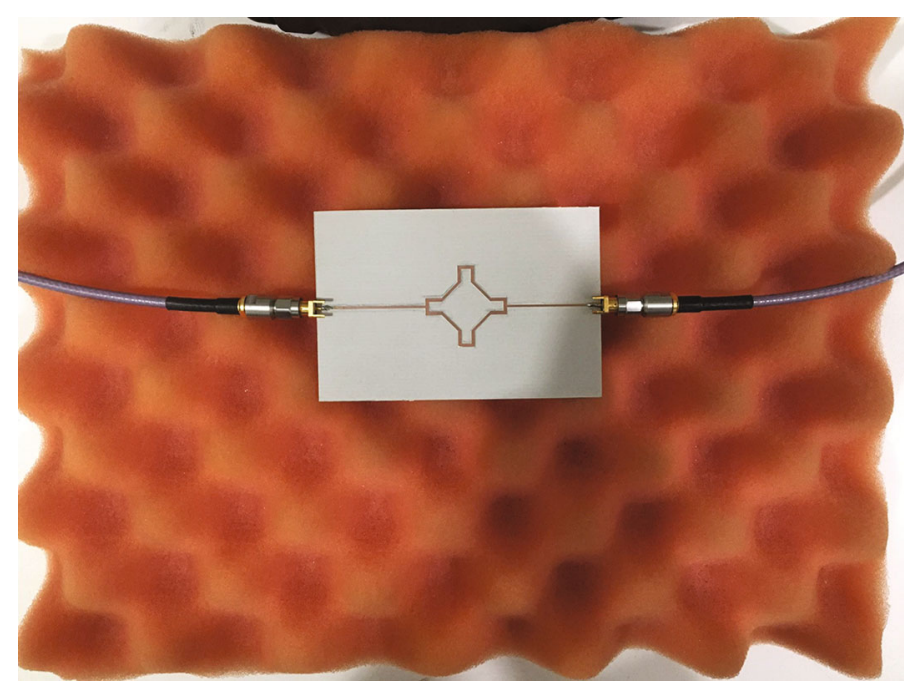

FIGURE 6: Fabricated sensor prototype.

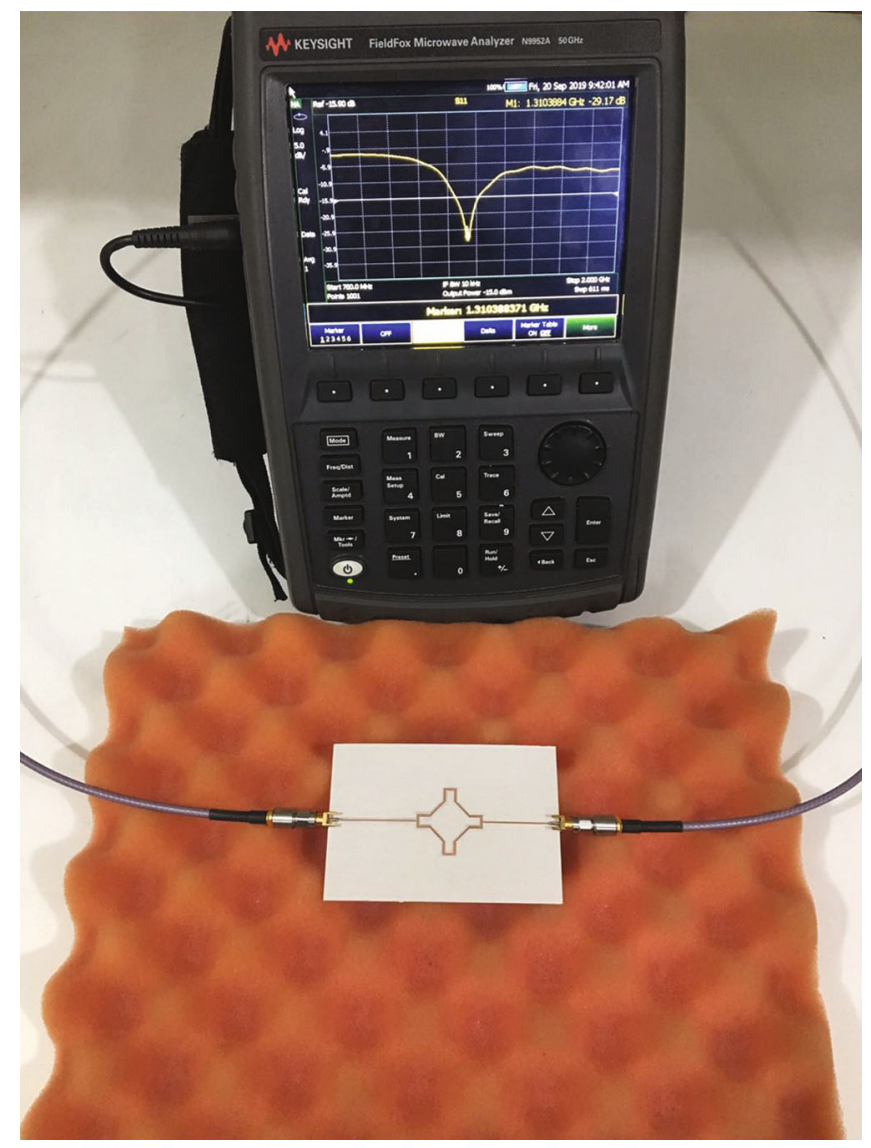

FIgURE 7: Experimental set-up (Sensor connected to the VNA).

permittivity values between 10 and 100 , since, with the frequency shift, the curves of the other resonances will overlap, providing an ambiguous response.

Figure 5 shows the first resonant frequency as a function of permittivity, with a variation between 10 and 100 . Since the permittivity range is large, to better describe the sensor's behavior, an exponential function is used. The sensitivity in this pole is low (23.1); however, since the resonance quality factor is high $(122.06,517.04,445.23$, and 535.87 for resonances $1,2,3$, and 4 , respectively), it is possible to extract sample permittivity.

Equation (9) provides a direct expression to calculate the permittivity of the sample material when knowing the resonant frequency of the sensor-sample. 


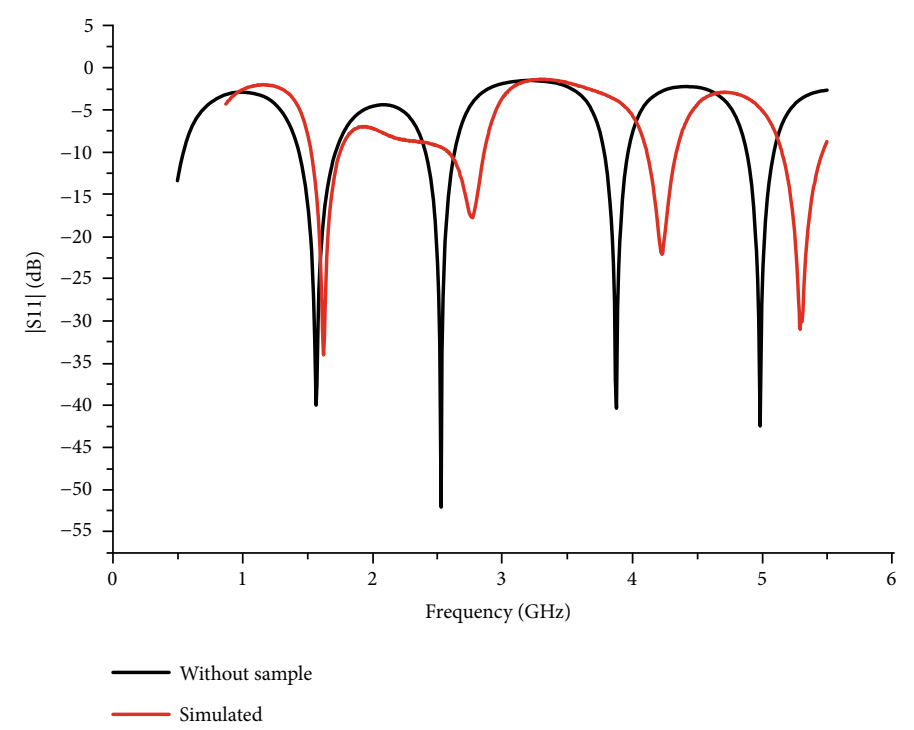

FIgURE 8: Comparison between simulated and experimental results.

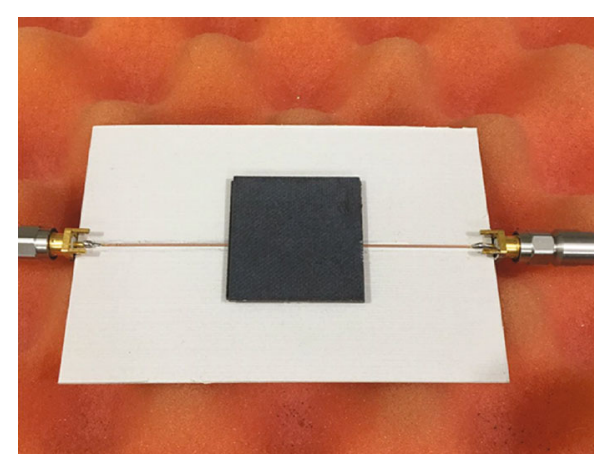

(a)

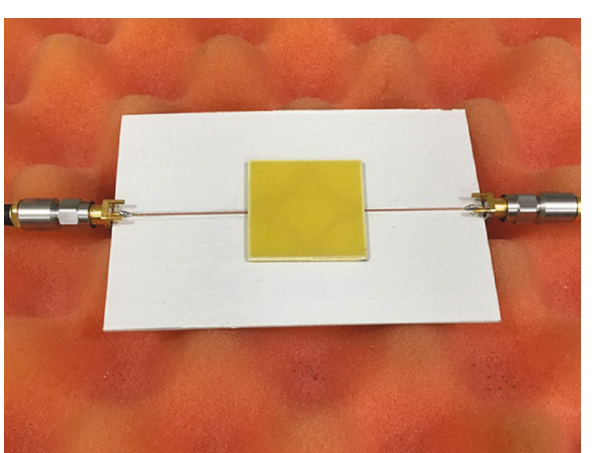

(b)

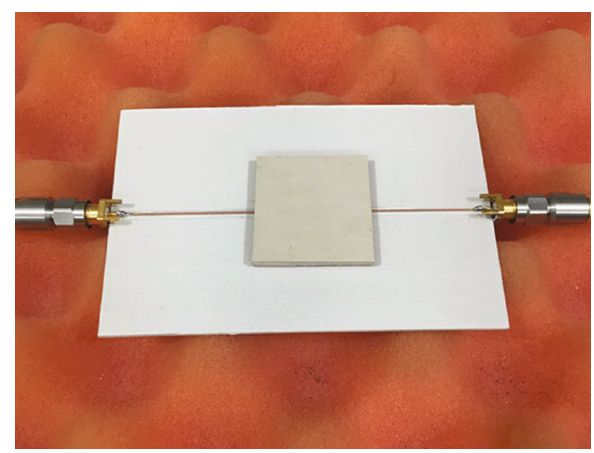

(c)

FIGURE 9: Sensor with samples on top: (a) Rogers 5880; (b) FR4; (c) AD1000.

For $\varepsilon_{r}$ from 10 to 100 :

$$
\varepsilon_{r}=3454.3 e^{-5,659 * f r_{3}},
$$

\section{Experimental Demonstration}

The sensor is manufactured using an Everprecision EP2006H prototyping machine and a Rogers ${ }^{\circledR} 3010$ substrate. Dimensions of the complete device are $9.95 \times 6.67 \mathrm{~cm}$. Experimental results are obtained using a Keysight N9952A VNA. Figure 6
TABLE 1: Material samples.

\begin{tabular}{|c|c|c|}
\hline Sample & Height (h) & $\varepsilon r$ \\
\hline RT/duroid ${ }^{\circledR} 5880$ & $1.27 \mathrm{~mm}$ & 2.2 \\
\hline FR-4 & $1.6 \mathrm{~mm}$ & 4.5 \\
\hline $\mathrm{AD} 1000^{\mathrm{TM}}$ & $3 \mathrm{~mm}$ & 10.2 \\
\hline
\end{tabular}




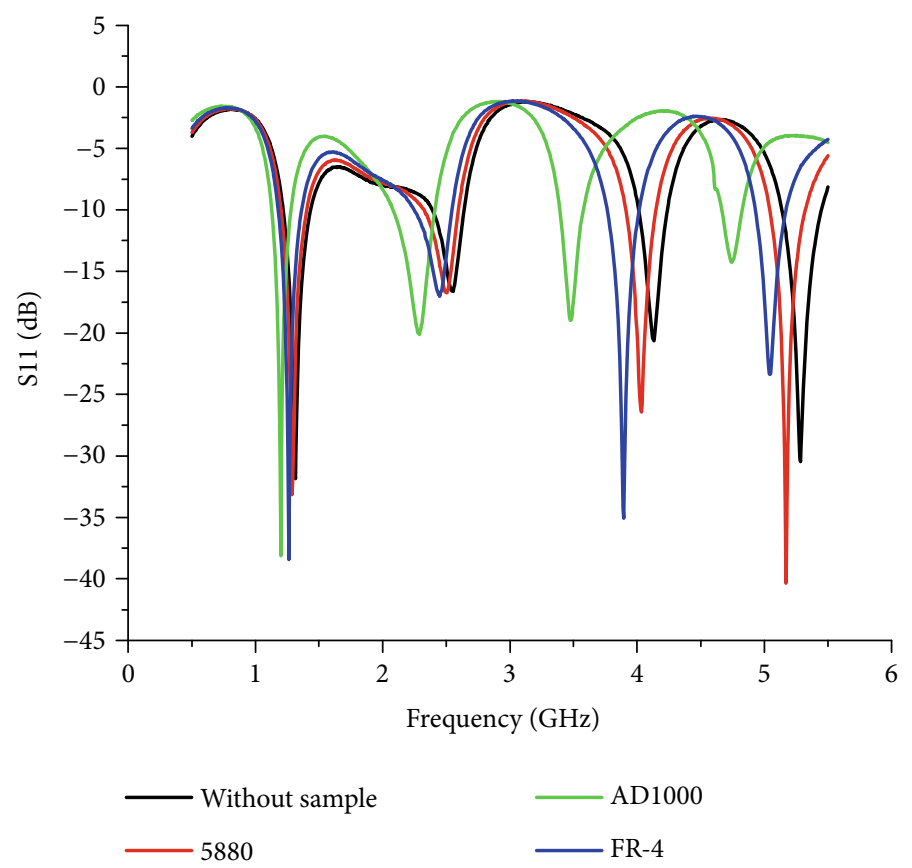

FIGURE 10: Reflection coefficient of the sensor for various samples.

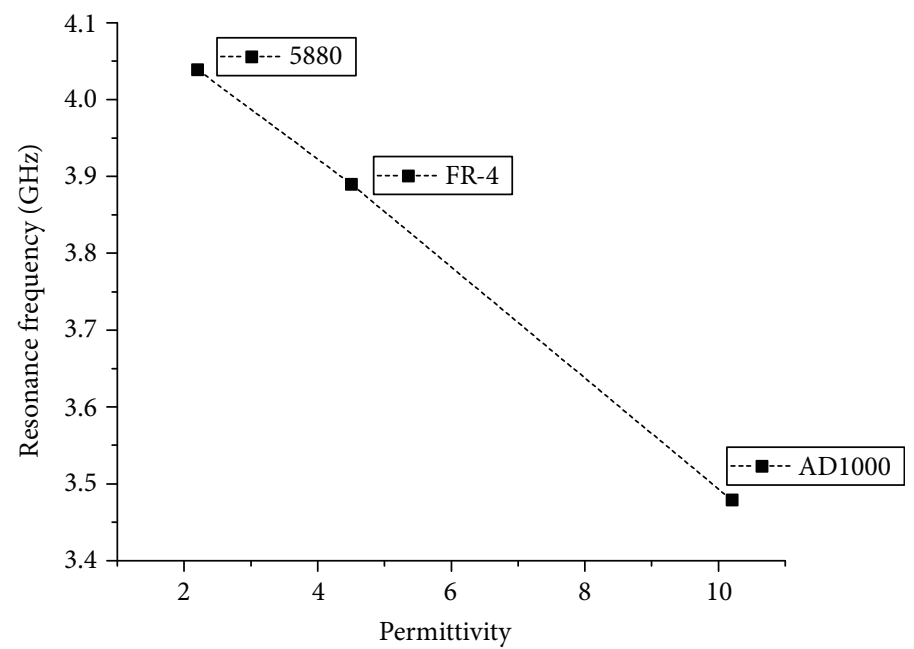

Figure 11: Sensitivity level of the sensor device for the well-known samples in Table 1.

shows the manufactured sensor prototype, and Figure 7 shows the measurement setup.

The comparison between experimental and simulated results for the sensor without sample is shown in Figure 8. The frequency shift observed on the poles of the frequency response may be due to fabrication tolerances; additionally, the SMA connector soldering process can cause inductive or capacitive effects at the input ports of the device under test.

To validate the sensor, dielectric substrates with well-known material permittivity are measured using the proposed sensor, as shown in Figure 9. Since the permittivity of the dielectric samples is in the range from 2.2 to 10.2 , the third pole is chosen to analyze the samples, thus, avoiding resonance overlap, and consequently, an ambiguous response. Table 1 shows the samples used with their respective characteristics.

The choice of pole for sensing depends on the sample measured. Since the permittivity of the dielectric samples are in the range from 2.2 to 10.2 , the third pole is chosen to analyze the samples, since it presents the best response in this frequency range of interest, compared to the other resonances, thus avoiding resonance overlap, and consequently, a possible ambiguous response.

As shown in Figure 10, the samples deposited on top of the sensor produce different magnitudes of the reflection coefficient $|\Gamma|$, when measured. When comparing the results with the sensor without samples, the results present resonant frequency shifts of 95,140 , and $415 \mathrm{MHz}$ for Rogers 5880, FR4, and 
TABLE 2: Comparison between different sensors.

\begin{tabular}{|c|c|c|c|c|c|}
\hline \multirow{2}{*}{ Reference } & \multirow{2}{*}{ Methods } & \multicolumn{4}{|c|}{ Specifications } \\
\hline & & Freq $(\mathrm{GHz})$ & Q-factor & S-parameter (dB) & Sensitivity \\
\hline$[20]$ & Circular split ring resonator & 3.68 & 409 & $-35.0\left(\mathrm{~S}_{21}\right)$ & 39.1 \\
\hline$[21]$ & Omega shape resonator* & 1.9 & 12 & $-6.0\left(S_{21}\right)$ & - \\
\hline [18] & Rectangular open-loop resonator & 2.13 & 143 & $-26.1\left(\mathrm{~S}_{11}\right)$ & 48.8 \\
\hline This work (third pole) & Sierpinski fractal curve resonator & 4.13 & 445.23 & $-20.6\left(S_{11}\right)$ & 70.3 \\
\hline
\end{tabular}

*Estimated.

AD1000, respectively. The experimental sensitivities obtained are $11.3,27.1,70.3$, and 53 , for resonances $1,2,3$, and 4 , respectively.

The sensor resonant frequency changes according to the permittivity of the samples, thus, it is possible to make a linear fit as shown in Figure 11, which shows the permittivity versus resonant frequency curve, hence, the permittivity of the sample can be calculated or predicted, according to the following equation.

$$
\varepsilon_{r}=-14,197 f r_{3}+59,619
$$

In Table 2, a comparison between the proposed multiband Sierpinski fractal curve sensor (third pole) with other resonator-based sensors available in the literature is provided.

\section{Conclusion}

This paper presents a sensor design developed for the characterization of materials whose permittivity ranges from 1 to 100. The use of the Sierpinski curve geometry to form a microstrip resonator resulted in high sensitivity of the projected sensor, with a direct relationship with the capacitive area of fractal resonator structure. Through the sensitivity curve of the sensor, it is possible to obtain the permittivity of other samples, without the aid of new software simulations.

The sensor design has four resonance frequencies, resulting in a multiband device for material characterization. Any of the four resonant frequencies of the sensor provides a high sensitivity to measure samples in the range from 1 to 10.2 , being experimentally demonstrated by measuring samples with known permittivity values using the third pole to measure the samples. The first resonance is suitable to measure samples in the permittivity range from 10 to 100 since the other bands will overlap in this permittivity range and may result in ambiguities. Therefore, the proposed sensor is useful to measure samples over a large range of permittivity values.

\section{Data Availability}

The first author has all data.

\section{Conflicts of Interest}

The authors declare that they have no conflicts of interest.

\section{Acknowledgments}

This work has been supported by the science and innovation ministry of Spain through project RTI2018-099841B-I00. Part of this work has been supported by the Generalitat de Catalunya under grant 2017 SGR 891.

\section{References}

[1] J. G. D. Oliveira, E. N. M. G. Pinto, V. P. Silva Neto, and A. G. D’Assunção, "CSRR-based microwave sensor for dielectric materials characterization applied to soil water content determination," Sensors, vol. 20, no. 1, p. 255, 2020.

[2] J. Piotrowski, J. Skulski, A. Mikina, and B. Galwas, "Automated industrial system with resonator sensor for characterization of high-lossy dielectric materials," in 11th International Microwave Conference, Warsaw, Poland, May 1996.

[3] E. Nyffors and P. Vainikainen, "Industrial microwave sensors," in 10th International Microwave Conference, pp. 45-54, Ksiaz, Poland, May 1994.

[4] E. Reyes-Vera, G. Acevedo-Osorio, M. Arias-Correa, and D. E. Senior, "A submersible printed sensor based on a monopolecoupled split ring resonator for permittivity characterization," Sensors, vol. 19, no. 8, p. 1936, 2019.

[5] P. V. Vainikainen, Measurement Electronics of Industrial Microwave Resonator Sensors, [Ph.D. Thesis], Helsinki University of Technology, 1991.

[6] A. K. Kraszewski, "Microwave research in agriculture," in 10th International Microwave Conference, pp. 87-93, Ksiaz, Poland, May 1994.

[7] X. Zhang, C. Ruan, T. Haq, and K. Chen, "High-sensitivity microwave sensor for liquid characterization using a complementary circular spiral resonator," Sensors, vol. 19, no. 4, p. 787, 2019.

[8] J. Skulski and B. A. Galwas, "Planar resonator sensor for moisture measurements," in 12th International Conference on Microwaves and Radar, pp. 692-695, Krakow, Poland, May 1998.

[9] G. Bianconi, C. Pelletti, R. Mittra, K. Du, S. Genovesi, and A. Monorchio, "Spectral domain characteristic basis function method for efficient simulation of microstrip devices in layered media," IET Microwaves, Antennas \& Propagation, vol. 6, no. 4, pp. 411-417, 2012.

[10] M. Kent, "The use of strip-line configurations in microwave moisture measurement," Journal of Microwave Power, vol. 7, no. 3, pp. 185-193, 1972.

[11] M. G. Negri, Introdução ao estudo dos fracais, Federal University of Goiás, Thesis for the degree of Master of Technology, 2014. 
[12] E. W. Weisstein, "Sierpiski sieve. From MathWorld, A Wolfram Web Resource," https://mathworld.wolfram.com/ SierpinskiSieve.html.

[13] M. Amiri, F. Tofigh, N. Shariati, J. Lipman, and M. Abolhasan, "Miniature tri-wideband Sierpinski-Minkowski fractals metamaterial perfect absorber," IET Microwaves, Antennas \& Propagation, vol. 13, no. 7, pp. 991-996, 2019.

[14] H. M. Cundy and A. P. Rollett, Mathematical Models, Tarquin Publications, London, 1981.

[15] J. Feder, Fractals, Plenum Press, New York, 1988.

[16] K. Falconer, Fractal Geometry: Mathematical Foundations and Applications, John Wiley \& Sons, Chichester, 2004.

[17] R. K. Kanth, P. Liljeberg, H. Tenhunen, W. Ahmad, S. Shakya, and L. R. Zheng, "Autonomous use of fractal structure in low cost, multiband and compact navigational antenna," in 10th Mediterranean Microwave Symposium, pp. 135-138, Guzelyurt, Cyprus, August 2010.

[18] M. S. Coutinho, C. P. N. Silva, M. R. T. Oliveira, H. V. H. Silva Filho, G. G. Machado, and M. T. de Melo, "Planar sensor for powder grain characterisation," IET Microwaves, Antennas \& Propagation, vol. 12, no. 10, pp. 1666-1670, 2018.

[19] A. G. Neto, A. Gonçalves da Costa, and C. da Silva Moreira, "A new planar sensor based on the matryoshka microstrip resonator," in SBMO/IEEE MTT-S International Microwave and Optoelectronics Conference (IMOC), pp. 1-5, Aguas de Lindoia, Brasil, August 2017.

[20] N. Sharafadinzadeh, M. Abdolrazzaghi, and M. Daneshmand, "Investigation on planar microwave sensors with enhanced sensitivity from microfluidic integration," Sensors and Actuators A: Physical, vol. 301, article 111752, 2020.

[21] Y. I. Abdulkarim, L. Deng, M. Karaaslan et al., "Novel metamaterials-based hypersensitized liquid sensor integrating omega-shaped resonator with microstrip transmission line," Sensors, vol. 20, no. 3, p. 943, 2020. 